\title{
A Simple Optimization Workflow to Enable Precise and Accurate Imputation of Missing Values in Proteomic Datasets
}

Kruttika Dabke ${ }^{1,2}$, Simion Kreimer ${ }^{3}$, Michelle R. Jones ${ }^{1}$, and Sarah J. Parker ${ }^{3^{*}}$

${ }^{1}$ Center for Bioinformatics and Functional Genomics, Department of Biomedical Science, Cedars-Sinai Medical Center, Los Angeles, CA, 90048

${ }^{2}$ Graduate Program in Biomedical Sciences, Department of Biomedical Science, Cedars-Sinai Medical Center, Los Angeles, CA, 90048

${ }^{3}$ Advanced Clinical Biosystems Research Institute, Smidt Heart Institute, Departments of Cardiology and Biomedical Sciences, Cedars-Sinai Medical Center, Los Angeles, CA, 90048

\section{Correspondence:}

Sarah J Parker, PhD

Assistant Professor and Associate Director, Proteomics and Metabolomics Core Cedars-Sinai Medical Center

AHSP A9228

127 South San Vincente Boulevard

Los Angeles, CA 90048

Sarah.parker@cshs.org 


\section{Table of contents}

Suppl. Figure 1. Evaluation of imputation methods for dilution series data set at the peptide and protein level.

Suppl. Figure 2: Evaluation of the dilution series data set after imputation of missing values using Random Forest

Suppl. Figure 3. Pearson's correlation coefficient for replicates of the pilot data set by protein intensities before and after imputation of missing values.

Suppl. Figure 4. Patterns of missing values at the fragment level for beta-actin like protein and GAPDH before and after outlier fragment filtration.

Suppl. Figure 5. Patterns of missing values at the fragment level for PAX8 and MUC16 before and after outlier fragment filtration.

Suppl. Figure 6. Number of proteins with different proportions of missing values before and after mapDIA fragment filtration

Suppl. Figure 7. Strategy for imputation and evaluation of missing values

Suppl. Figure 8. Evaluation of dilution series data set with missing values and correlation of replicates for complete dilution series data set.

Supplementary Table 1. NAguideR results with different missingness thresholds for pilot data set both at the peptide and protein level.

Supplementary Table 2. Results of differential expression analysis before and after imputation (before outlier fragment filtration).

Supplementary Table 3. Results of differential expression analysis before and after imputation (after outlier fragment filtration) including permuted data set correlations.

Supplementary Table 4. KEGG enrichment analysis for statistically significant proteins upregulated in stromal samples (after outlier fragment filtration) 
A
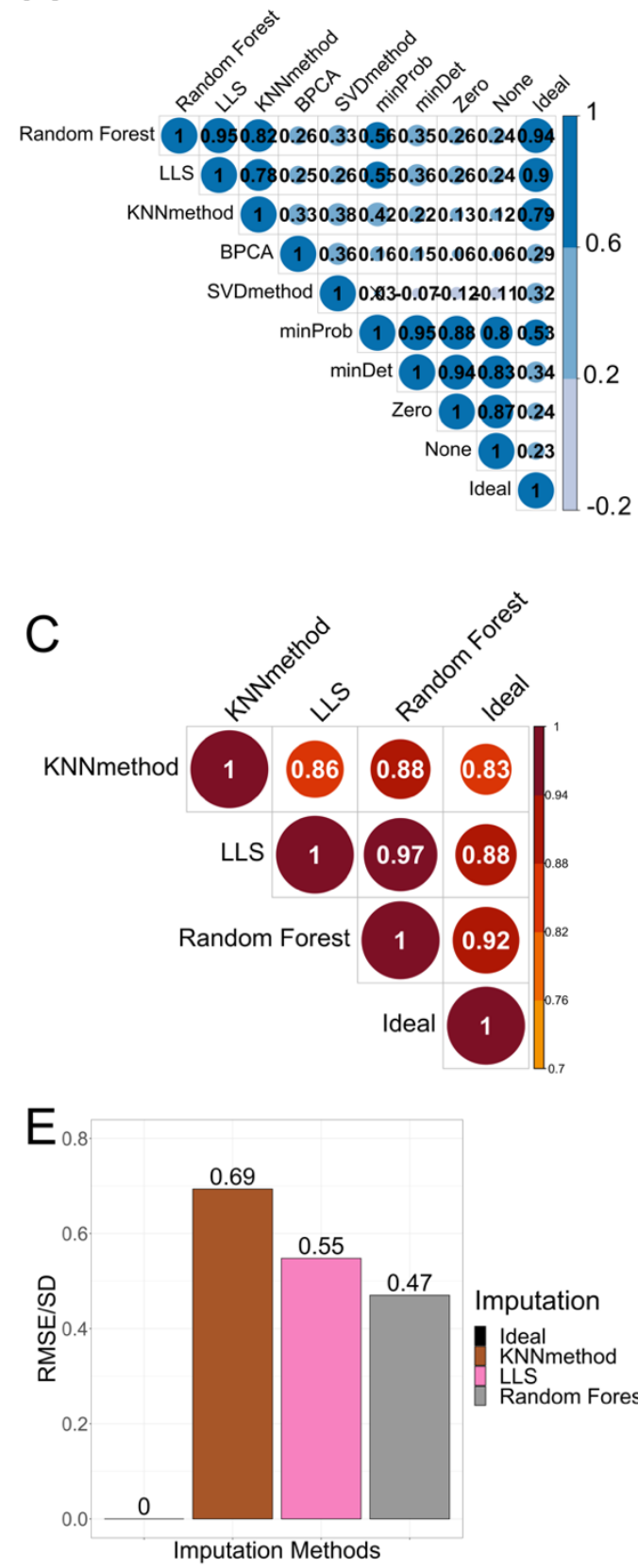

B

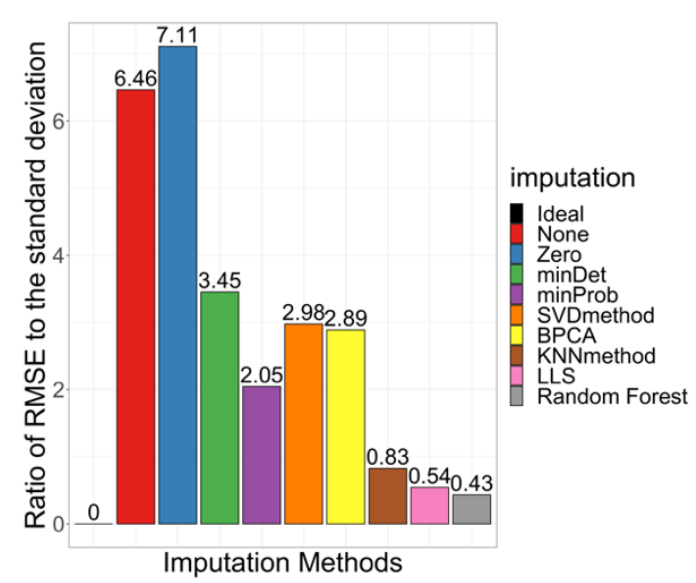

D

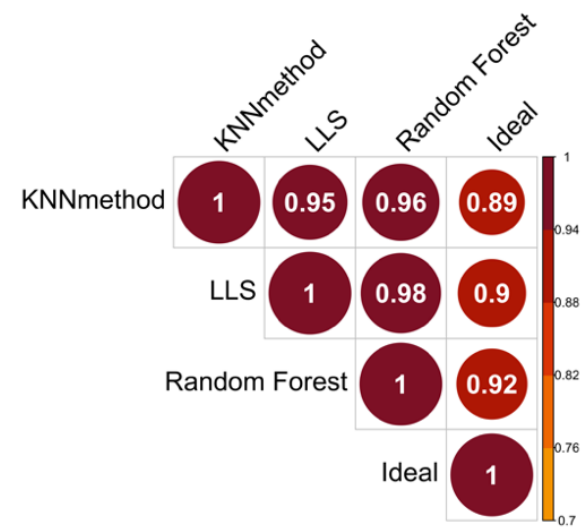

$\mathrm{F}$

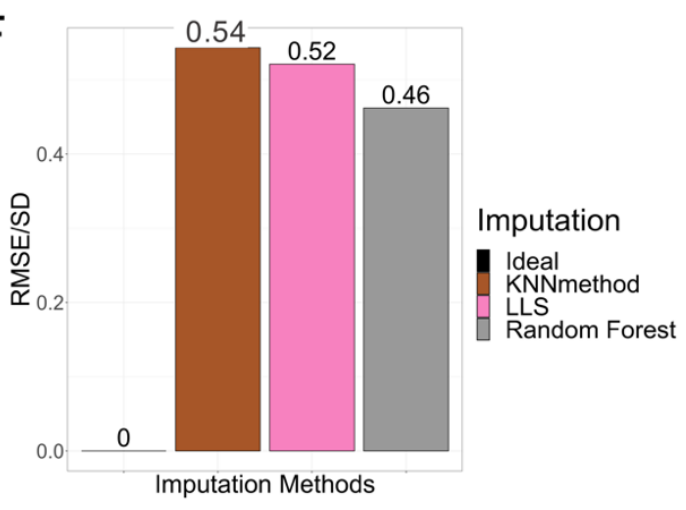

Supplementary Figure 1: Evaluation of imputation methods for dilution series data set at the peptide and protein level.

A) Pearson's correlation coefficient of fold changes shows high correlation with ideal fold change after LLS and RF imputation ( 0.9 and 0.94 respectively). B) Error estimation between ideal fold change and different imputation methods. C, D) Pearson's correlation coefficient for the top three ranked imputation methods after imputation at the peptide (C) and protein (D) level. E, F) Ratio of RMSE to S.D. for the top three ranked imputation methods after imputation at the peptide (C) and protein (D) level. 

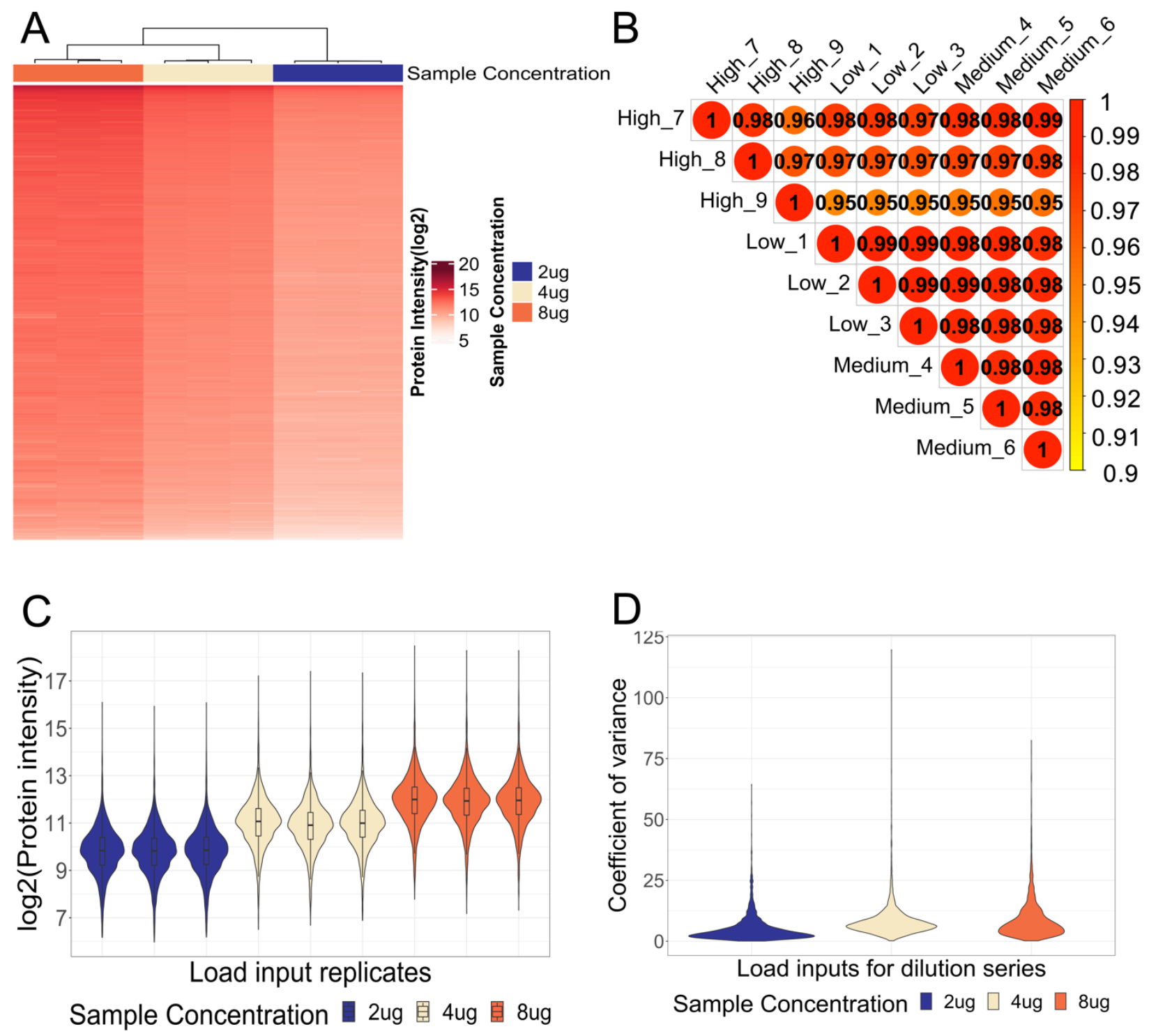

\section{Supplementary Figure 2: Evaluation of the dilution series data set after imputation of missing values using Random Forest}

A) Unsupervised hierarchical clustering of protein intensities (rows) shows clustering of samples (columns) by replicates. B) Pearson's correlation coefficient of replicates by protein intensities shows a high correlation among all samples. C) Distribution of protein intensities of replicates show a step wise increase in median protein intensity corresponding to load. D) \% Coefficient of variance (CV) calculated by protein intensities of the three replicates within each load. 

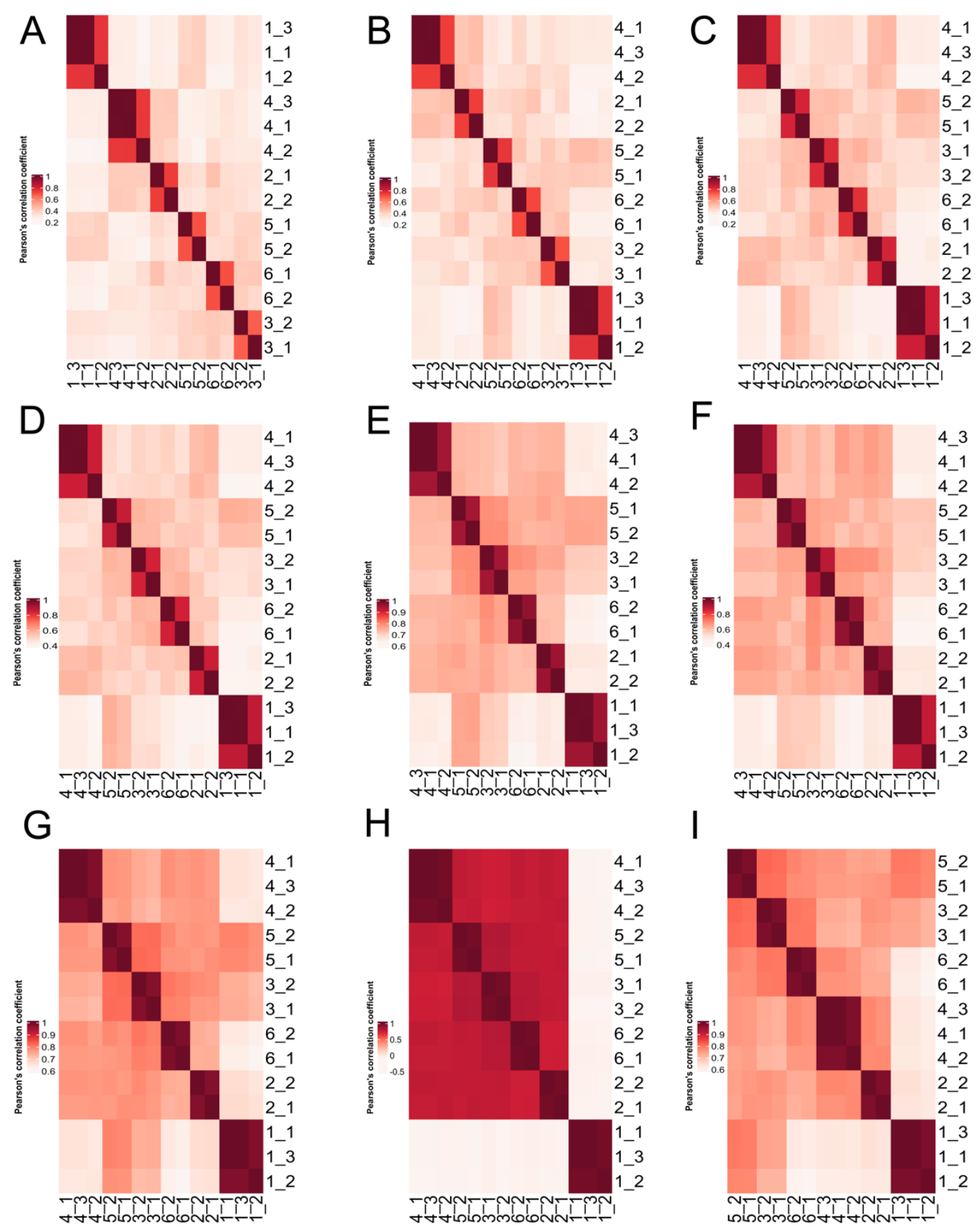

Supplementary Figure 3: Pearson's correlation coefficient for replicates of the pilot data set by protein intensities before and after imputation of missing values. A) Non imputed data set, B) Zero imputation, C) minDet imputation, D) minProb imputation E) k-NN imputation F) SVD imputation G) BPCA imputation H) LLS imputation I) Random Forest imputation 


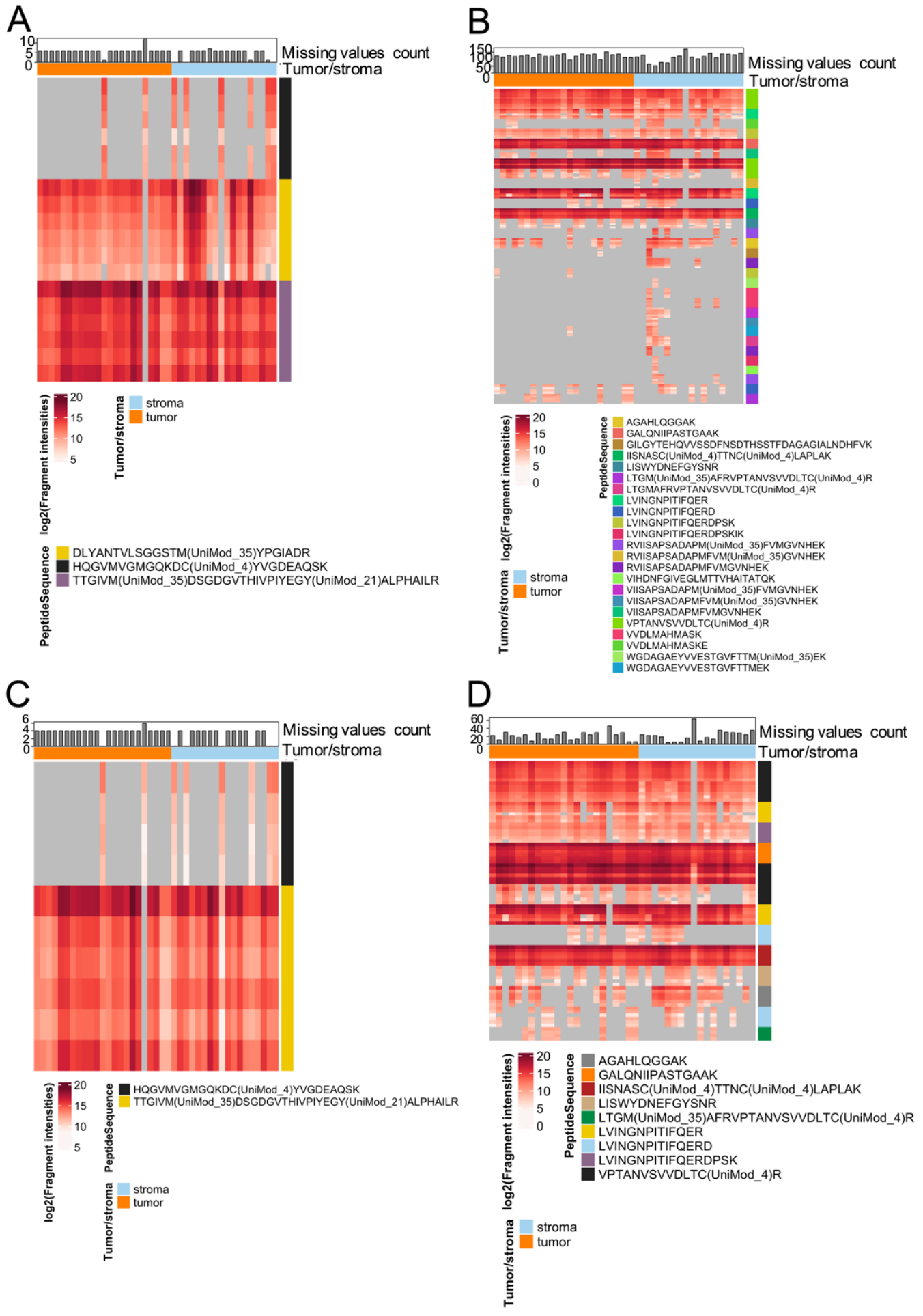

Supplementary Figure 4: Fragment level missingness pattern; beta-actin like protein and GAPDH

Fragment level missingness pattern before outlier fragment filtration for beta-actin like protein (A) and GAPDH (B)

Fragment level missingness pattern after outlier fragment filtration for beta-actin like protein (C) and GAPDH (D) 
A
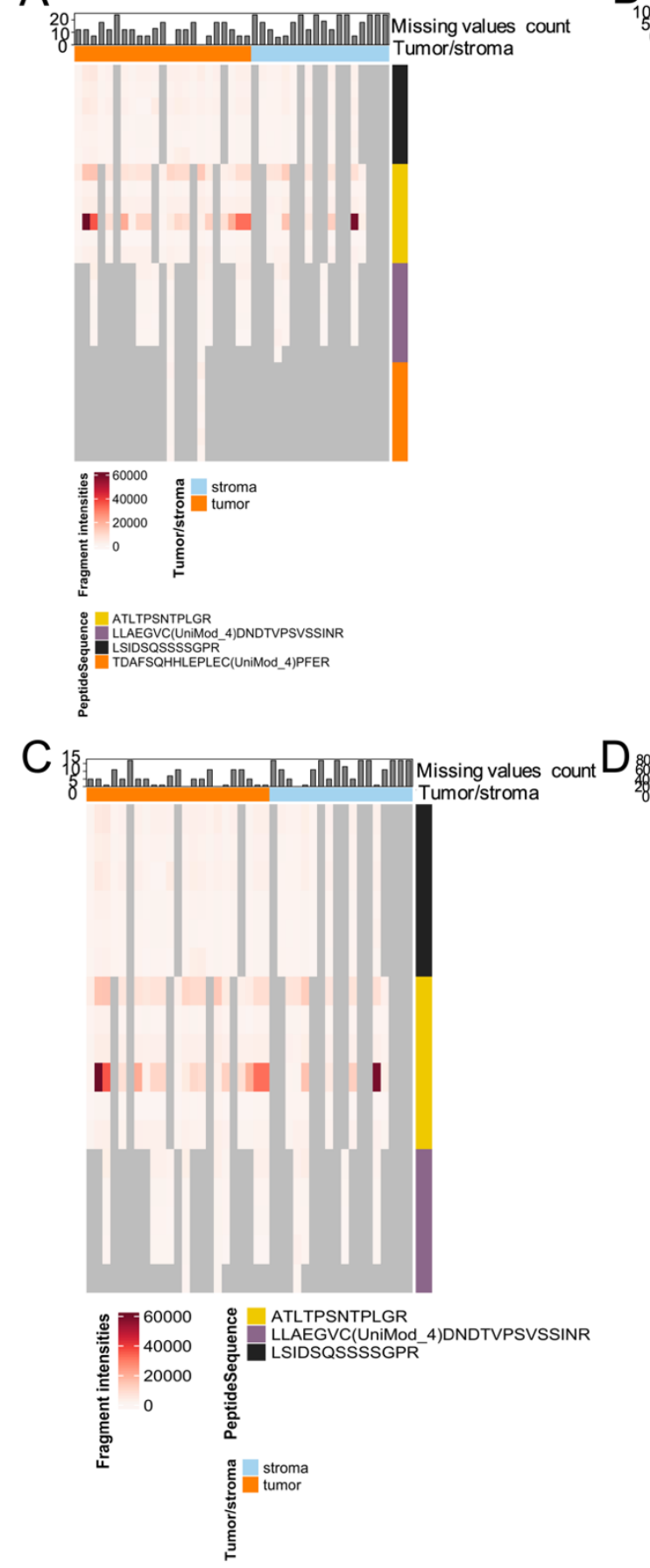

$\mathrm{B}$
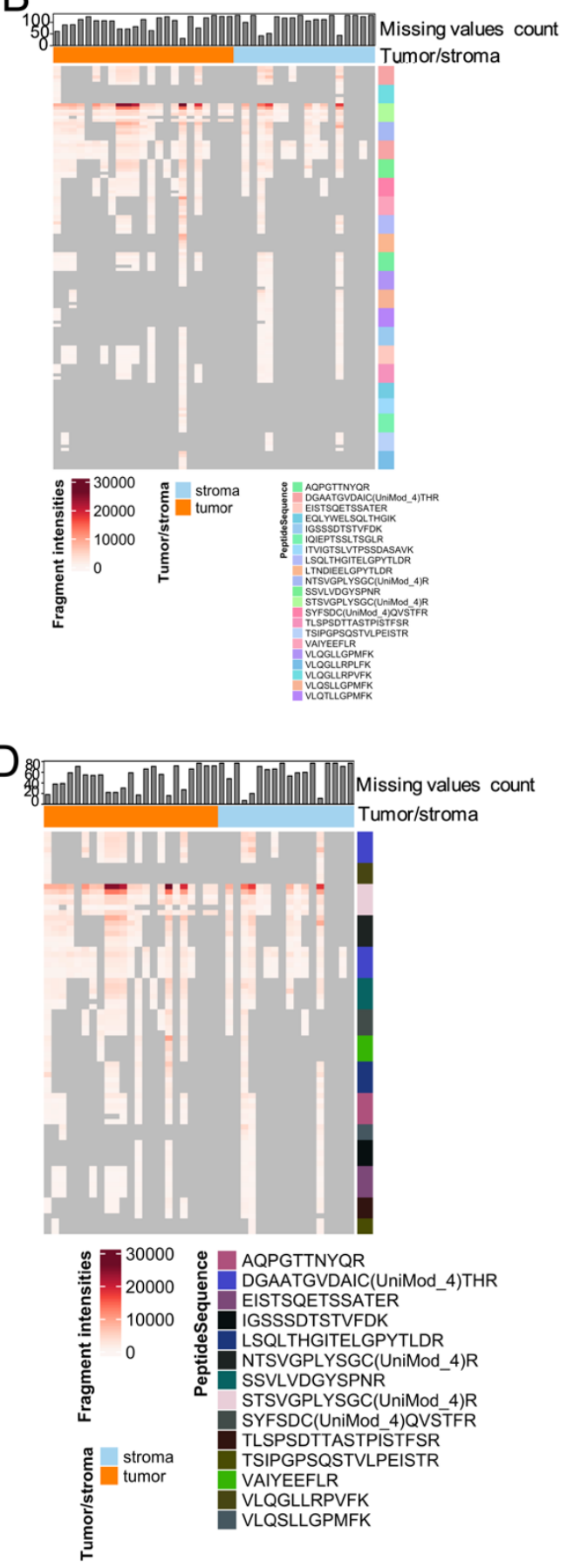

Supplementary Figure 5: Fragment level missingness pattern; PAX8 and MUC16 Fragment level missingness pattern before outlier fragment filtration for PAX8 (A) and MUC16 (B)

Fragment level missingness pattern after outlier fragment filtration for PAX8 (C) and MUC16 (D) 

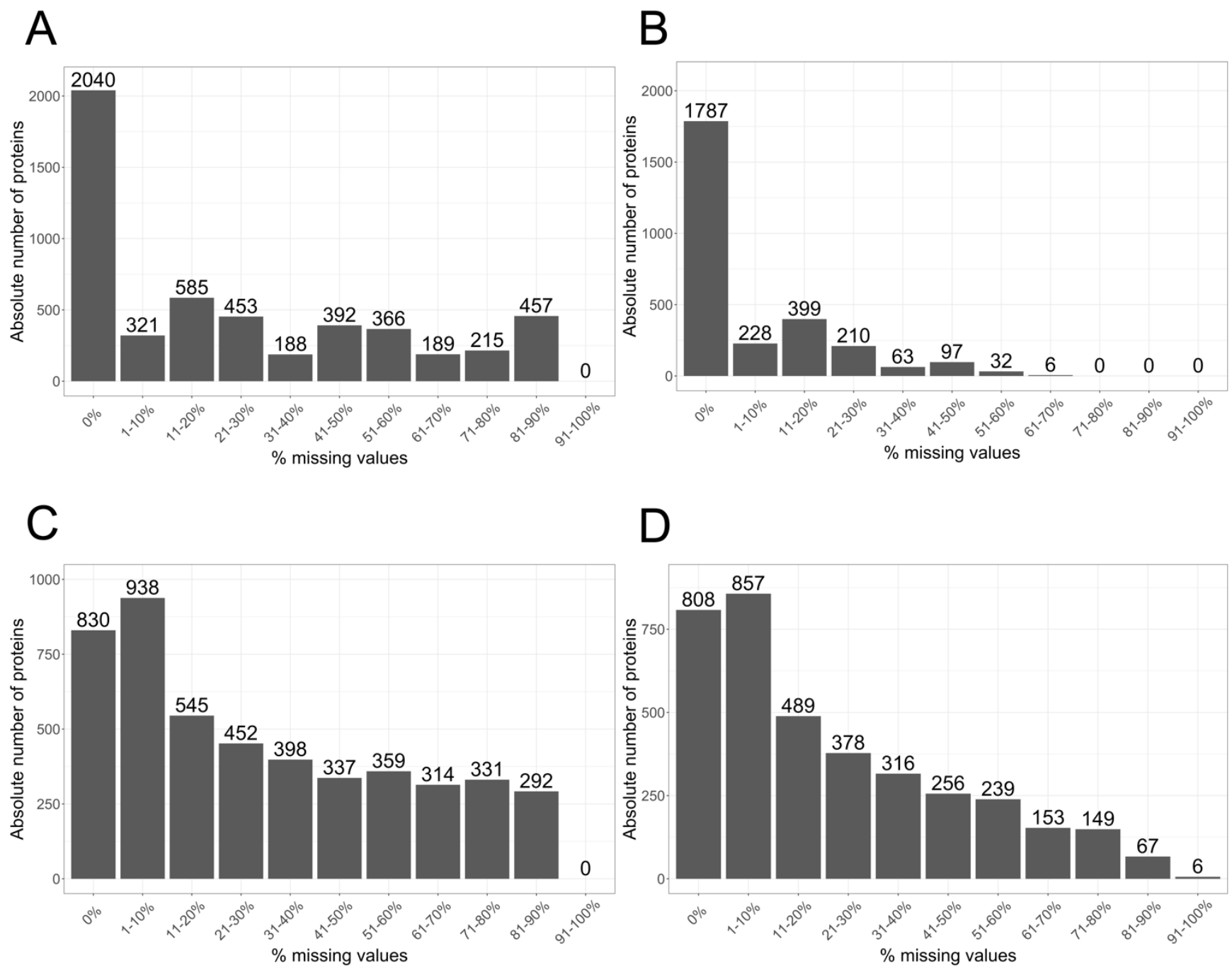

Supplementary figure 6: Number of proteins with different proportions of missing values before and after mapDIA fragment filtration

Distribution of proteins with different proportions of missing values for the pilot data set before filtration of outlier fragments (A) and after filtration of outlier fragments (B). Distribution of proteins with different proportions of missing values for the clinical proteomic data set before filtration of outlier fragments (C) and after filtration of outlier fragments (D). 
A

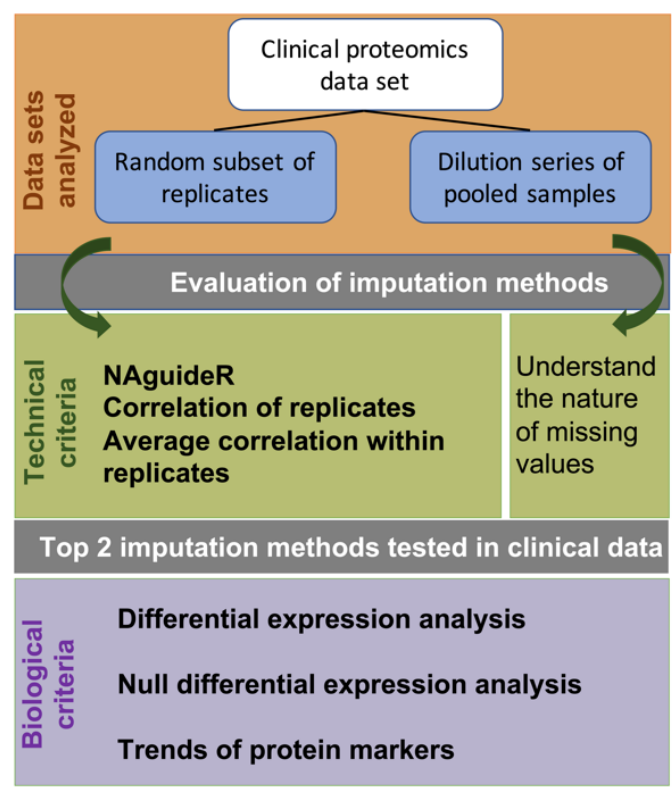

B

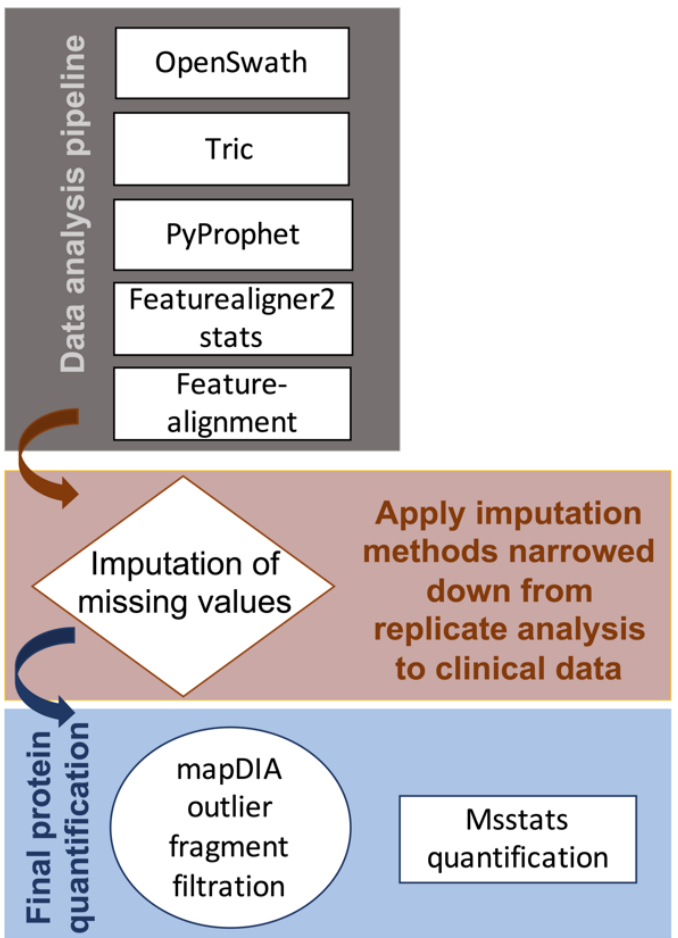

C

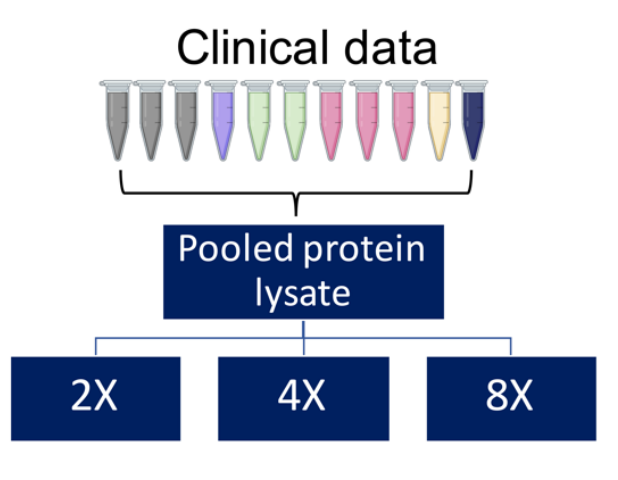

Dilution series data set

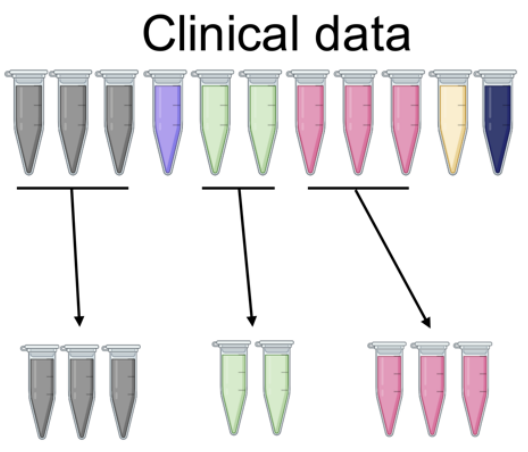

Pilot data set

Supplementary Figure 7: Strategy for imputation and evaluation of missing values A) Flow chart outlining strategy for evaluation of imputation methods for clinical proteomic data sets. B) Workflow for data analysis and imputation of missing values. C) Data sets used in the analysis 
A

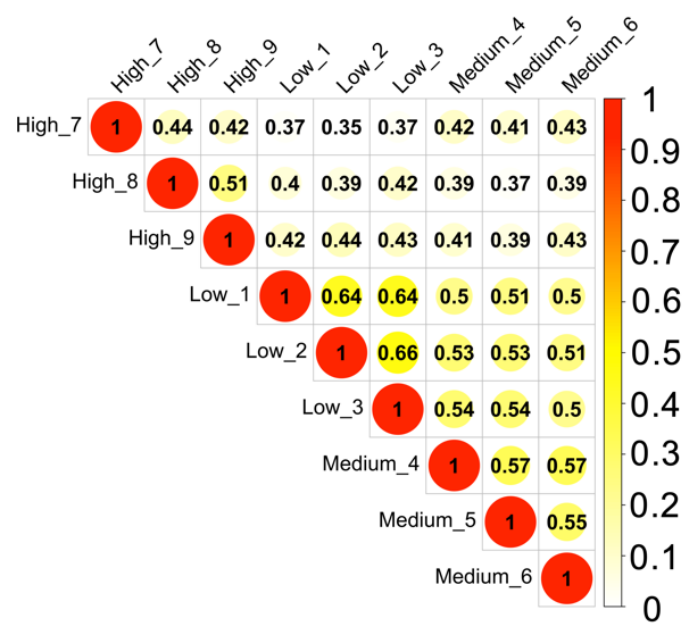

B

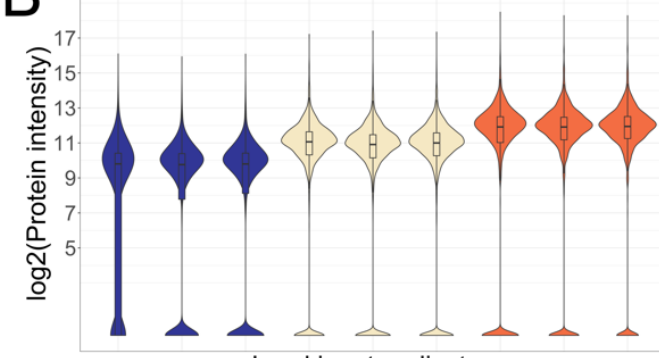

Load input replicates
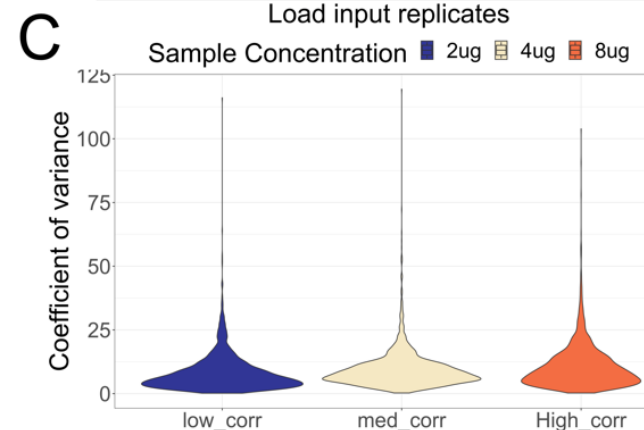

Load inputs for dilution series

Sample Concentration $\square$ 2ug $\square$ ug $\square$ 8ug

D
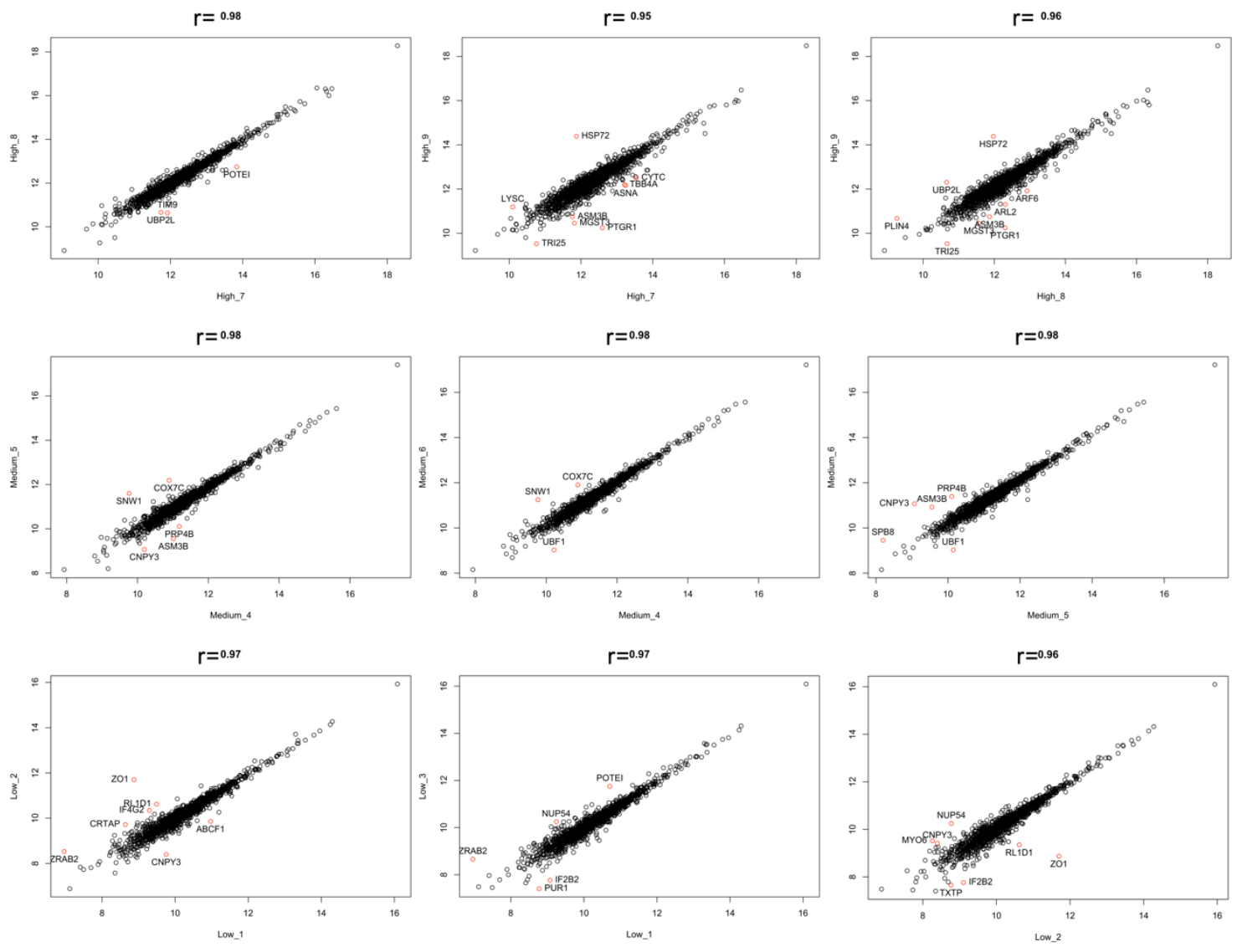
Supplementary figure 8: Evaluation of dilution series data set with missing values and correlation of replicates for complete dilution series data set.

A) Pearson's correlation coefficient of replicates by protein intensities for data set with missing values. B) Median protein intensities of replicates for data set with missing values. (missing values were converted to 0 for $A$ and B) C) \% Coefficient of variance (CV) calculated by protein intensities of the 3 replicates within each load for data set with missing values. D) Scatter plots of log 2 protein intensities of paired replicates for each sample concentration in complete dilution series data set without missing values (proteins that showed more than one log fold change difference between replicates of each sample were highlighted in red and labelled, $r=$ Pearson's correlation coefficient). 